\title{
Niobates Nanowires: Synthesis, Characterization and Applications
}

\author{
Rachel Grange ${ }^{1}$, Fabrizia Dutto ${ }^{2}$ and Aleksandra Radenovic ${ }^{2}$ \\ ${ }^{1}$ Friedrich Schiller University Jena \\ ${ }^{2}$ Ecole Polytechnique Fédérale de Lausanne \\ ${ }^{1}$ Germany \\ 2Switzerland
}

\section{Introduction}

Perovskite oxides such as alkaline niobates crystal possess many interesting properties including piezoelectricity, pyroelectricity, electro-optic and nonlinear optical response (Bhalla et al., 2000). The most common alkaline niobates material is lithium niobate $\left(\mathrm{LiNbO}_{3}\right)$. Since the discovery of $\mathrm{LiNbO}_{3}$ ferroelectricity (Matthias \& Remeika, 1949), its properties are widely exploited by electronic devices particularly in telecom applications (Wooten et al., 2000). Those devices are made from bulk or thin films material and serves as sensors, actuators, detectors or filters. Potassium niobate $\left(\mathrm{KNbO}_{3}\right)$ is most known for its large nonlinear coefficients ideal for wavelength conversion like second-harmonic generation (SHG), sum frequency mixing, as material in optical parametric oscillator, or lead-free piezoceramics (Saito et al., 2004). Sodium niobate $\left(\mathrm{NaNbO}_{3}\right)$, less studied than $\mathrm{LiNbO}_{3}$ and $\mathrm{KNbO}_{3}$, also belongs to the alkaline niobates. Generally associated with potassium, $\mathrm{NaNbO}_{3}$ is a very promising lead-free piezoelectric ceramics (Guo et al., 2004).

Besides bulk and thin films structures, zero- (0D) and one-dimensional (1D) alkaline niobates nanostructures were synthesized recently to combine the dimensional confinement with the other known properties of perovskite materials. Different synthesis routes have been explored to obtain OD nanoparticles or nanoflakes from alkaline niobates such as mechano-chemical milling (Kong et al., 2008; Schwesyg et al., 2007), nonaqueous route (Niederberger et al., 2004), sol-gel method (L. H. Wang et al., 2007) or hydrothermal route (An et al., 2002). Almost simultaneously, anisotropic alkaline niobates 1D structure were synthesized with various methods such as template assisted pyrolysis resulting in regular arrays of tubes (Zhao et al., 2005), solution-phase synthesis resulting in rod-like structures (Wood et al., 2008), or hydrothermal route giving free-standing nanowires with high aspect ratio (Magrez et al., 2006).

Up to now, the nanomaterials properties have been well characterized using standard materials sciences methods like X-ray diffraction (XRD), scanning electron (SEM) or transmission electron (TEM) microscopy. However, nonlinear optical or electro-optic properties have been rarely studied. Moreover, few applications have used these types of nanowires while combining the various physical properties of perovskite alkaline materials and the anisotropic shape at the nanoscale level. Nanometric SHG light probe manipulated by optical tweezers and capable of guiding light has been already demonstrated (Nakayama 
et al., 2007), as well as localized SHG light source in optofluidics environment (Grange et al., 2009).

In this chapter, we focus on the synthesis, optical characterization and applications of Li-, $\mathrm{Na}-, \mathrm{KNbO}_{3}$ nanowires. Some crystal and second-order optical properties of the investigated alkaline niobates are summarized in Table 1 . We will describe hydrothermal and molten salt synthesis. We will measure some optical and physical properties of these nanowires. Then we propose the use of plasmonics gold nanoshells to enhance the SHG signal and for best biocompatibility with biological applications. Finally we show interesting applications using optical tweezers and microfluidics chips by combining dielectrophoresis and SHG.

\begin{tabular}{|c|c|c|c|}
\hline Material & Crystal system & $\mathrm{d}_{\mathrm{eff}}(\mathrm{pm} / \mathrm{V})$ & References \\
\hline $\mathrm{LiNbO}_{3}$ & Trigonal & {$[2-34.4]$} & $\begin{array}{l}\text { (Boyd, 2008; Fluck \& Gunter, 2000; Shoji et al., } \\
\text { 2002; Sutherland, 2003; Weber, 2003) }\end{array}$ \\
\hline $\mathrm{KNbO}_{3}$ & Orthorhombic & [10.8-19.6] & $\begin{array}{c}\text { (Fluck \& Gunter, 2000; Shoji et al., 2002; } \\
\text { Weber, 2003) }\end{array}$ \\
\hline $\mathrm{NaNbO}_{3}$ & Orthorhombic & {$[0.8-4.5]$} & (Johnston et al., 2010; Ke et al., 2008) \\
\hline
\end{tabular}

Table 1. Typical crystal system at room temperature and its corresponding second-order susceptibility tensor elements $\left(\mathrm{d}_{\mathrm{eff}}\right)$ range.

\section{Synthesis methods}

Several research groups developed different chemical synthetic approaches to fabricate crystalline alkaline niobates nanowires such as exploiting sol-gel route (Pribosic et al., 2005), hydrothermal route (An et al., 2002; Magrez et al., 2006; H. F. Shi et al., 2009; G. Z. Wang, Selbach et al., 2009; G. Z. Wang, Yu et al., 2009; Wu et al., 2010) and molten salt synthesis (MSS) (Li et al., 2009; Santulli et al., 2010).

Among the three studied alkaline niobate materials, $\mathrm{LiNbO}_{3}$ nanowires synthesis is the most challenging one. So far only two groups reported successful synthesis of free-standing $\mathrm{LiNbO}_{3}$ (Grange et al., 2009; Santulli et al., 2010). In contrast multiple synthesis routes can be found for other alkaline materials as potassium niobate $\mathrm{KNbO}_{3}$ (Li et al., 2009; Magrez et al., 2006; Nakayama et al., 2007; G. Z. Wang, Selbach et al., 2009; G. Z. Wang, Yu et al., 2009) and sodium niobate $\mathrm{NaNbO}_{3}$ (Li et al., 2009; H. F. Shi et al., 2009; Wu et al., 2010) nanowires. We have investigated two chemical synthesis methods for the nanowires fabrication: hydrothermal synthesis and molten salt synthesis (MSS).

Hydrothermal synthesis is a technique used to crystallize substances under moderate temperatures $\left(200-250^{\circ} \mathrm{C}\right)$ and high pressures. Thanks to the use of an autoclave, a thickwalled steel cylinder with an hermetic seal, the wanted crystalline materials can be obtained in one step (Fig. 1 a). The large amount of material that can be fabricated, the easiness (one step synthesis) and the speed of the synthesis make this approach very convenient. To optimize the synthesis parameters one can adjust the temperature, the time, the pressure (by an external pressure or the degree of the autoclave filling), the caustic soda concentration, the solid-liquid ratio and additives to control the properties of the end product. Thus, hydrothermal synthesis is promising because many operation parameters can be modulated to control the particle size and morphology. Due to its simplicity the hydrothermal technique has been widely studied and employed in inorganic synthesis for many years. 
Molten salt synthesis (MSS) is used to prepare complex oxides from their constituent oxide. It is a multi steps synthesis and it is way longer than hydrothermal synthesis approach but more adaptable to other alkaline materials than $\mathrm{KNbO}_{3}$ as shown in the literature (Li et al., 2009; Santulli et al., 2010). Oxides corresponding to a perovskite compound are mixed with one or two kinds of salt sand then heated at a temperature above the melting point of the salt to form a flux of the salt composition (Fig. $1 \mathrm{~b}$ ). At this temperature, the oxides are rearranged and then diffused rapidly in a liquid state of the salt. With further heating, particles of the perovskite phase are formed through the nucleation and growth processes.

A huge advantage of chemical synthesis in respect to chemical vapor deposition (CVD) or lithographic fabrication of nanowires is the ability to synthesize free-standing nanowires. Thus no further step is needed to isolate or detached the nanowires, because no substrate is involved in the synthesis.
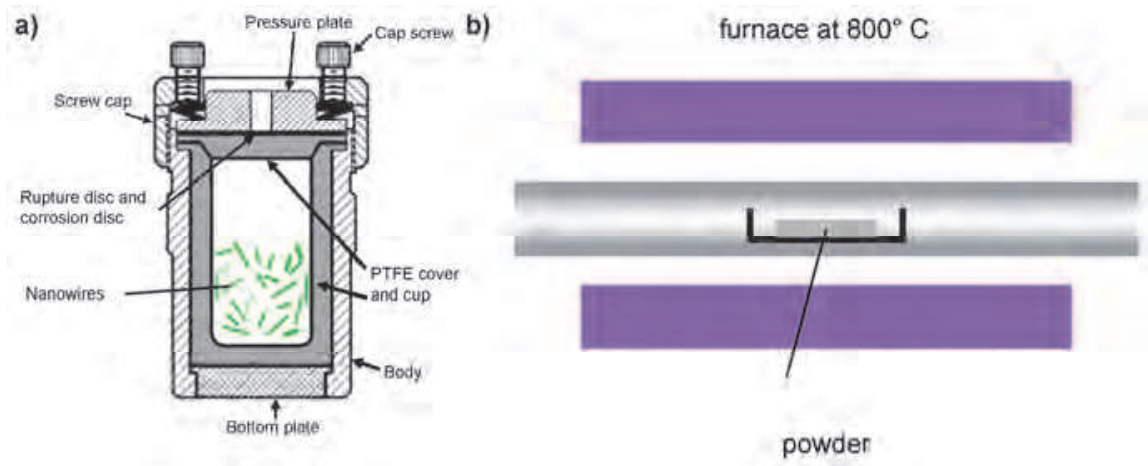

Fig. 1. a) Schematic diagram of a typical laboratory autoclave from Parr (Acid digestion bomb, 125ml, Model 4748). b) Schematic of the experimental setup for molten salt synthesis.

Fig. 2 shows typical results of $\mathrm{KNbO}_{3}$ nanowires hydrothermally synthesized following (Magrez et al., 2006) recipe, $\mathrm{NaNbO}_{3}$ nanowires hydrothermal synthesized following (Zhu et al., 2006) recipe and $\mathrm{LiNbO}_{3}$ nanowires molten salt synthesized following (Santulli et al., 2010) recipe. Due to the low solubility of lithium hydroxide, molten salt synthesis is preferred to fabricate $\mathrm{LiNbO}_{3}$ nanowires even if it is a multistep synthesis.
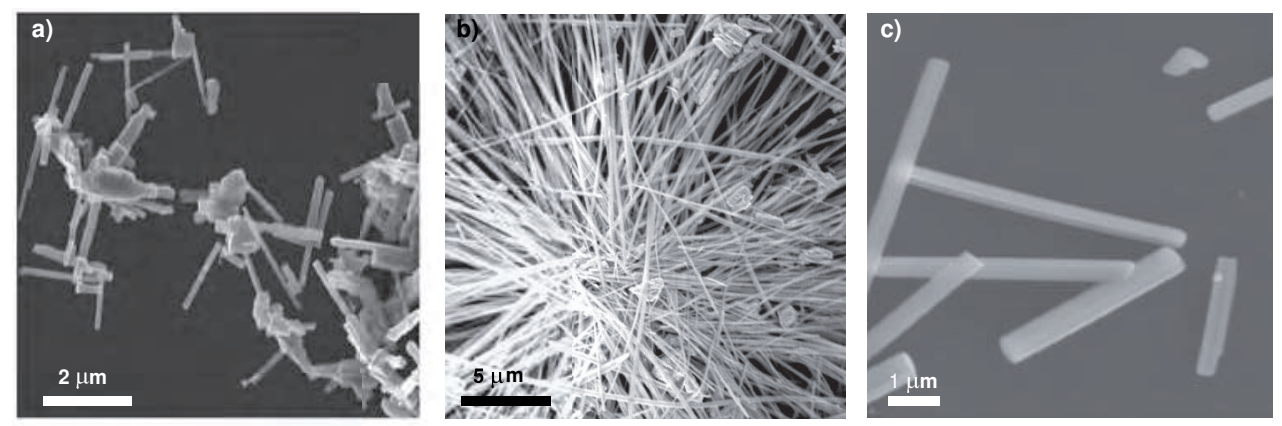

Fig. 2. SEM images of a) $\mathrm{KNbO}_{3}$ nanowires with an aspect ratio up to 25. b) $\mathrm{NaNbO}_{3}$ nanowires with an aspect ratio up to $50 . \mathrm{c}) \mathrm{LiNbO}_{3}$ nanowires with an aspect ratio up to 10 . 


\section{Material properties and characterization}

To investigate the structure of produced nanowires XRD characterization was employed, confirming the $\mathrm{KNbO}_{3}$ orthorhombic structure in a percentage of $100 \%, \mathrm{NaNbO}_{3}$ orthorhombic phase in a percentage of $15 \%$ and $\mathrm{LiNbO}_{3}$ trigonal phase in a percentage of $75 \%$ (see Fig. 3 a to c).

a)

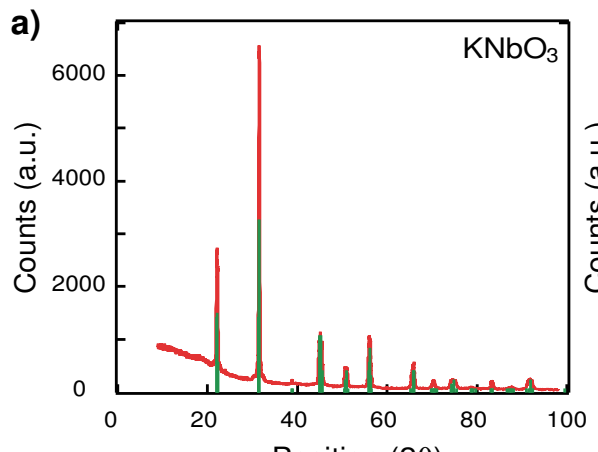

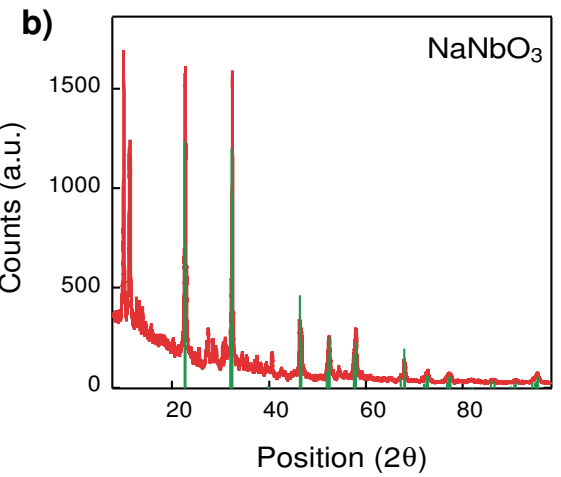

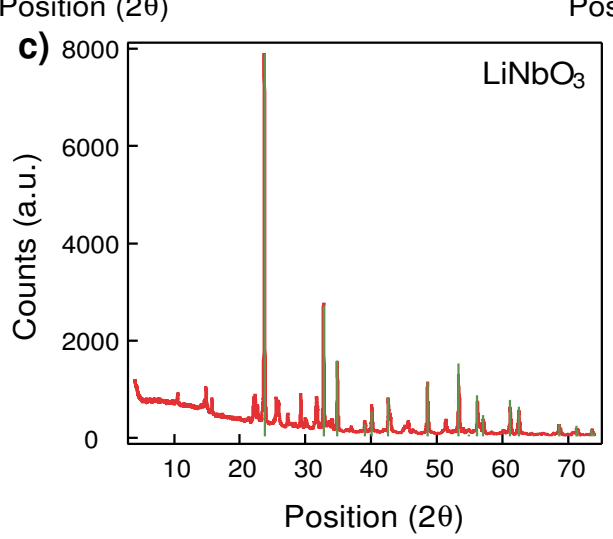

Fig. 3. X-ray diffraction (XRD) measurements (red) together with theoretical peaks (green) expected for desired phases of a), $\mathrm{KNbO}_{3}$ b) $\left.\mathrm{NaNbO}_{3}, \mathrm{c}\right) \mathrm{LiNbO}_{3}$.

In the hydrothermal synthesis procedure, to increase the percentage of preferred nanowire crystal structure, a calcination step (annealing at high temperatures such as $550^{\circ} \mathrm{C}$ ) can be performed. This calcination step increases the amount of ordered material phase in respect to other phases present in the sample, without changing the shape of the sample (Ke et al., 2008; H. Shi et al., 2009). For $\mathrm{NaNbO}_{3}$ synthesis, this additional step resulted in the drastic increase in the amount of pure material switching from $15 \%$ to $64 \%$ of presence of $\mathrm{NaNbO}_{3}$ ordered phase in the sample (Fig. $3 \mathrm{~b}$ ).

Besides XRD, that will give results over a high number of nanowires, it is possible to perform Raman scattering measurements on single nanowires that allow to distinguish materials and reveal unexpected phenomena (Louis et al.). As a preliminary result, we performed Raman measurements on $\mathrm{KNbO}_{3}$ nanowires and for comparison we show $\mathrm{LiNbO}_{3}$ nanoflakes Raman measurements too (Fig. 4). Typical $\mathrm{LiNbO}_{3}$ traces show peaks between $300 \mathrm{~cm}^{-1}$ and $480 \mathrm{~cm}^{-1}$ 
(Santulli et al., 2010). $\mathrm{KNbO}_{3}$ has one strong peak right below $300 \mathrm{~cm}^{-1}$ and nothing else between 300 and the silicon substrate peak (Louis et al.). The spectra are then different enough even between two alkaline niobates to easily distinguish the materials.

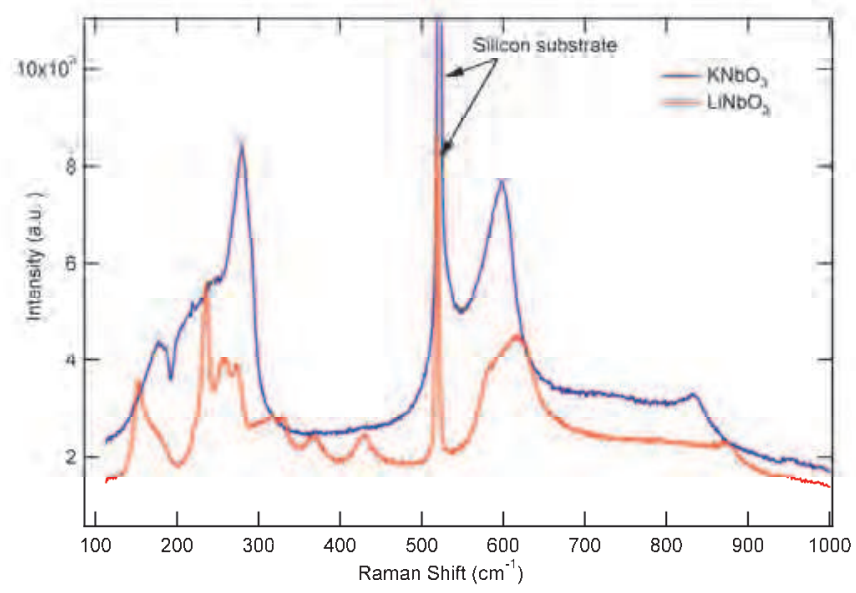

Fig. 4. Raman spectrum of $\mathrm{KNbO}_{3}$ nanowires (blue) at $80 \mathrm{~K}$ showing the typical shape known for this material and of $\mathrm{LiNbO}_{3}$ nanoflakes (red).

\section{Nonlinear optical characterization}

The perovskite nanowires with their non centrosymmetric crystal structures exhibit secondorder optical effects which open up a wide range of applications even the nanoscale level. Indeed, this nonlinear effect scales with the square of the electric field, but it is a volume effect which is measureable down to a single nanoparticle level even in far field microscopy (Hsieh et al., 2010), contrary to weak surface SHG effect of centrosymmetric materials (Dadap, 2008; Dadap et al., 1999). In the nonlinear regime, the optical response is expressed by the polarization $P$ as a power series in the electric field $E$ as

$$
\vec{P}=\varepsilon_{0} \chi_{1} \vec{E}+\varepsilon_{0} \chi_{2} \vec{E}^{2}+\varepsilon_{0} \chi_{3} \vec{E}^{3}+\ldots
$$

where $\varepsilon_{0}$ is the permittivity of free space and $\chi_{i}$ is the $\mathrm{i}$ th-order nonlinear optical susceptibility tensor. Each $\chi_{i}$ represents a different optical effect that can be summarized as follows for a physical understanding of Eq. (1). $\chi_{1}$, the linear susceptibility, is related to absorption and reflection of light. $\chi_{2}$ encompasses sum and difference frequency generation such as SHG. $\chi_{3}$ describes multiphoton absorption, third harmonic generation or coherent anti-Stokes Raman scattering.

Our applications are related to SHG as illustrated in Fig.5 a and b. When a nanocrystal of non centrosymmetric structure is optically excited at a fundamental frequency, it emits the optical signal at the exact doubled frequency. Only materials with crystalline structures lacking a centre of symmetry are capable of efficient SHG. Not only SHG will be scattered but the fundamental frequency too, thus efficient filters are needed to cut the fundamental. 

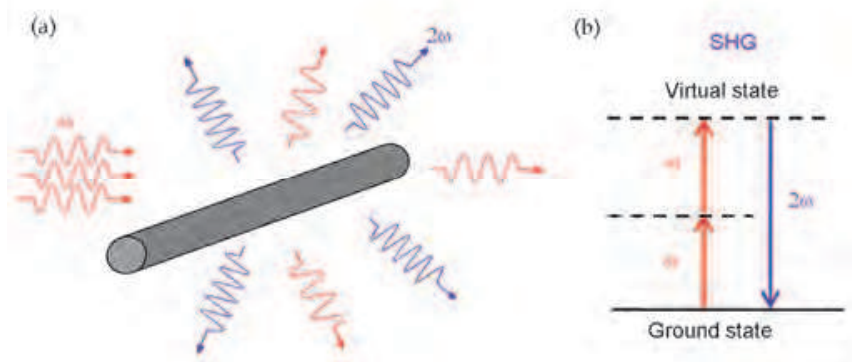

Fig. 5. (a) Schematic diagram of the SHG mechanism. (b) Energy diagram of the physical SHG mechanism.

The setup for the optical characterization of the SHG signal is shown in Fig. 6. A near infrared laser light (100 fs Ti:Sa oscillator) is focused onto a sample by lens L1 and the objective (OBJ) collects the signal imaged through a 4 f configuration with lens L2. Filters are used to cut the fundamental frequency and detect only a narrow band around the SHG frequency onto an electron multiplying charges coupled device (EMCCD).

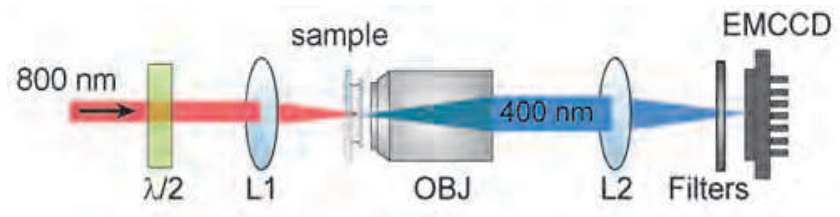

Fig. 6. Setup for measuring SHG from nanowires dried on a microscope glass slide. L1, L2, lenses; $\lambda / 2$ half wave plate; OBJ, objective.

Typical SHG measurements at different incident polarization angle are displayed in Fig.7. When the polarization is parallel to the nanowire the signal is the strongest, which is expected for a nanowires with an optical axis along the nanowire. Full polar SHG characterization is then possible and a fit of the experimental data can confirm the crystal orientation of a nanowire, which is not a priori known for chemically bottom-up synthesized nanowires (Grange et al., 2009).
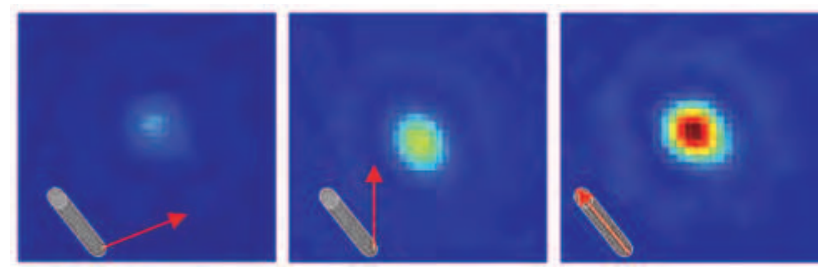

Fig. 7. EMCCD images of a single niobate nanowire under different incident light polarization. The insets sketched the wire position and the incident light polarization (red arrow). Images size $6 \times 6 \mu \mathrm{m}$.

In addition to conventional measurements of SHG from niobate nanowires we have carried out SHG characterization by using Optical Tweezers (OT) (see Fig.8). An OT uses a focused 
laser beam to provide an attractive or repulsive force (typically on the order of $\mathrm{pN}$ ), depending on the refractive index mismatch, to physically hold and move microscopic dielectric objects. Optical tweezers are routinely used tools in both physical and life sciences for manipulating objects from micron to the atomic scale and as force transducers in the $\mathrm{pN}$ range (Neuman \& Block, 2004).

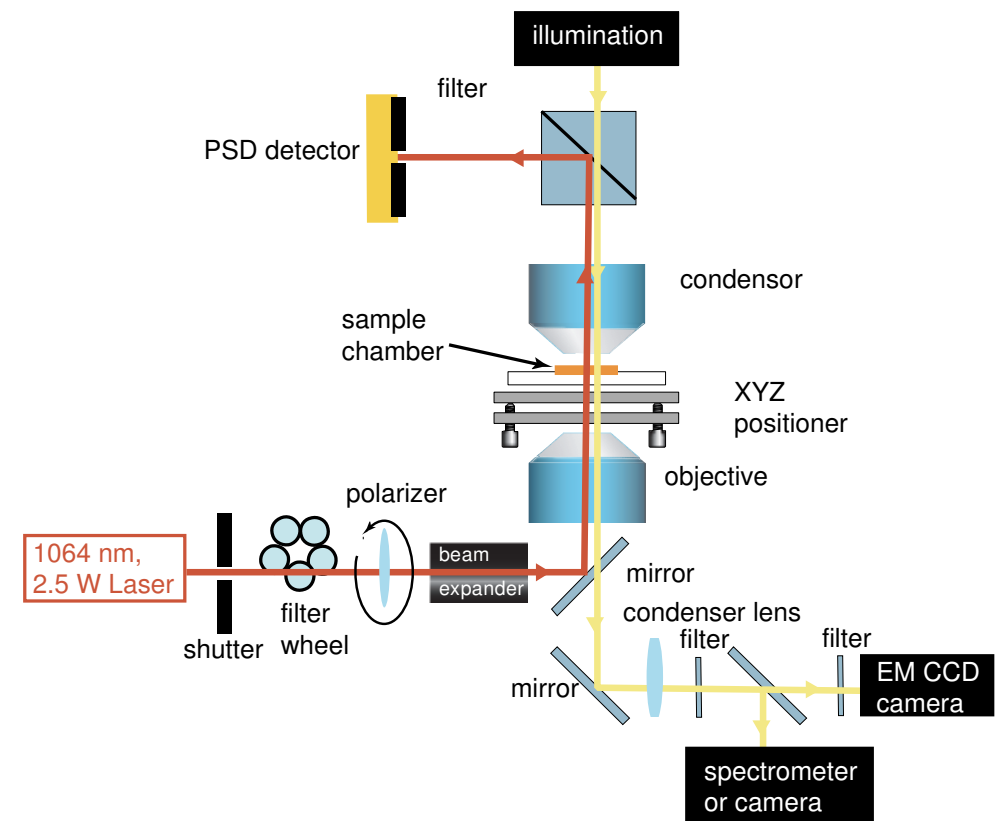

Fig. 8. Single beam optical tweezers setup for SHG characterization of the trapped nanowires. A Nd: $\mathrm{YVO}_{4} \mathrm{CW} 1064$ laser is used simultaneously for nanowire trapping and pumping. As in the conventional SHG setup, the signal is recorded using an EMCCD camera. After passing through the condenser, the output IR beam passes through an aperture and it is detected by a position sensitive detector (PSD) positioned at the rear focal plane of the condenser. Any transverse motion of the trapped nanowire will cause the center of the output beam to shift sideways on the PSD.

The ability to control and monitor the position of a mesoscopic object with nanometric precision is important for the rapid progress of nanoscience and it is perfectly suited for the study of biological phenomena. Besides its application in biology, OT are also an appealing tool for semiconductor nanowire integration owing to their ability to act in situ in closed aqueous chambers, their potential applicability to a broad range of dielectric materials, their spatial positioning accuracy $(<1 \mathrm{~nm})$, and the degree to which their intensity, wavelength and polarization can be controlled using tuneable lasers. All trapping experiments and subsequent SHG characterization were carried out on a home-built single beam optical tweezers system.

As in the conventional setup, we can probe SHG from individual niobate nanowire under different incident light polarization. The SHG signal under different incident polarization is much less sensitive in this orientation due to the tweezing geometry (Fig. 9). However, it 
may still slightly differ from wire to wire due to the variability of crystalline phases of the bottom-up synthesized nanowires.
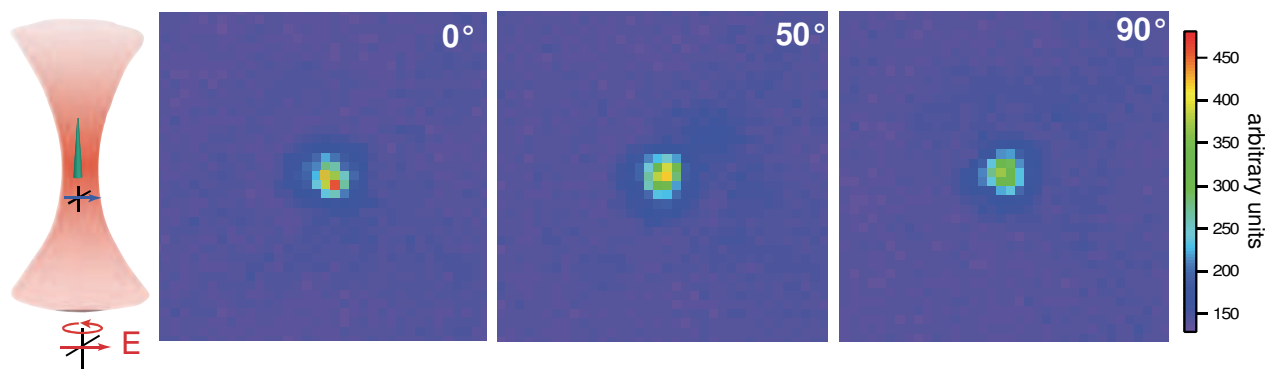

Fig. 9. EMCCD images of trapped single niobate nanowire under different incident light polarization. Images size $10 \times 10 \mu \mathrm{m}$.

\section{Plasmonics nanoshells for enhanced SHG}

We showed that non centrosymmetric nanowires exhibit SHG signal. However, nonlinear optical processes such as harmonic generation are generally inefficient at very small scale (the intensity goes down with the square of the particle volume). By developing nonlinear optical plasmonics core shell cavities, it was possible to strongly enhanced the SHG response of $\mathrm{BaTiO}_{3}$ nanoparticles (Pu et al., 2010). Similarly, $\mathrm{KNbO}_{3}$ nanowires are covered by a thin layer of gold to reach a near infrared plasmonics resonance and enhance the SHG signal for optimized used as imaging probes or localized light source.

(a)
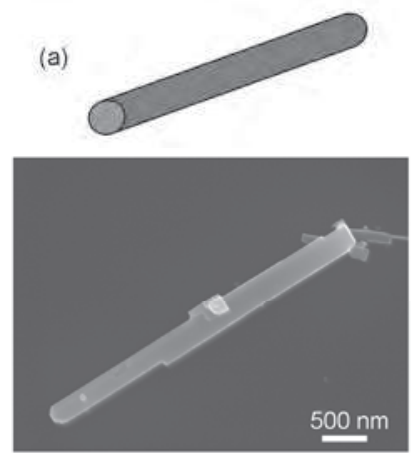

(b)
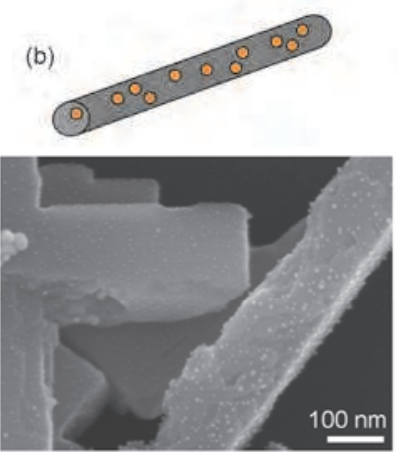
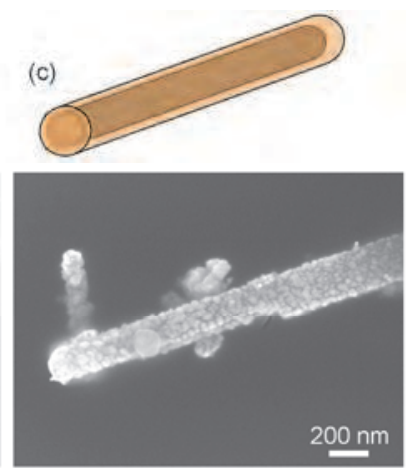

Fig. 10. Synthesis steps of $\mathrm{KNbO}_{3} / \mathrm{Au}$ core-shell nanowires with their corresponding SEM images. (a) bare $\mathrm{KNbO}_{3}$ nanowires (b) surface seeding with 2-3 nm colloidal gold particles (c) growth of a gold complete shell around the wires.

The gold coating process for $\mathrm{KNbO}_{3} / \mathrm{Au}$ core-shell nanowires involved three main steps illustrated in Fig. 10. First of all, primary amines are coated on the surface of the $\mathrm{KNbO}_{3}$ nanowires (Hsieh et al., 2009). The amino complex (Aminopropyltriethoxysilane) presents $\mathrm{NH}_{2}$ complex at the surface of the wires. Then, 2-3 nm gold particles are adsorbed onto the surface of $\mathrm{KNbO}_{3}$ wire thanks to the silane-amine functionalization ( $\mathrm{Au}^{3+}$ cations are 
attracted by the primary amine $\mathrm{NH}_{2}$ which become secondary amine) (Fig. $10 \mathrm{~b}$ ). Finally, the $\mathrm{Au}$ shell is grown all around the particle from the seeded particles by a reaction of reduction of gold by hydroxylamine (Fig. $10 \mathrm{c}$ ).

The gold reduction process to coat the wire surface still needs some improvement for a better and more uniform coverage of the surface. However, inhomogeneities may even enhance the plasmonics effects as commonly used in photovoltaic devices based on plasmonics nanoparticles (Atwater \& Polman, 2010). Further measurements will be performed to compare SHG signal from coated and uncoated wires.

\section{Applications of optically tweezed nanowires}

Recently, Nakayama et al. proved that several different types of nanowires can be stably trapped in optical traps; and that optical traps can thus be used to manipulate assembled three-dimensional nanowire heterostructures. Individual optically trapped nanowire can be placed and held in direct contact with living cells; in addition, one class of nanowires ( $\mathrm{KNbO}_{3}$ wires) exhibits efficient SHG and act as frequency converters, raising the possibility for a new type of scanning light microscopy (Nakayama et al., 2007) (Fig.11). Due to their small cross-section, nanowires represent ideal probes for mechanical and optical stimulation of cells and even organelles without overloading the sample with photons.
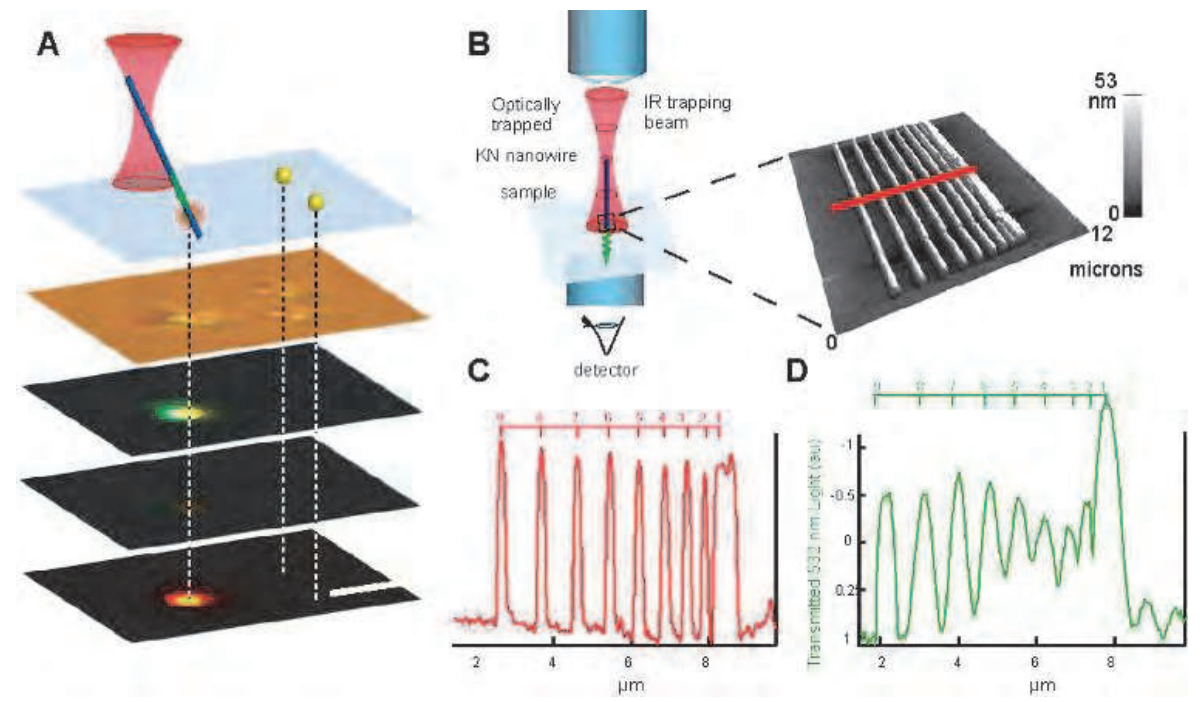

Fig. 11. Schematic of the experimental geometry where laser tweezers are used to accurately position the nanowire and excite fluorescence (A) Excitation of a fluorescent bead by waveguided SHG signal from an optically trapped $\mathrm{KNbO}_{3}$ nanowire. (B) Schematic of inverted optical scanning configuration band AFM topographic image of thermally evaporated pattern of gold stripes on a glass coverslip. (C) AFM line scan from region indicated in B). (D) Optical transmission profile captured by scanning a single $\mathrm{KNbO}_{3}$ nanowire over the metallic surface structure. The nanowire dimensions used to create the transmission line scan was measured by AFM: width=122 nm, length $=1.4 \mu \mathrm{m}$ and height=53 nm. From (Nakayama et al., 2007). 
The nanowires can be also used as complementary biomarkers for long term cell tracking experiments. Two important nanowire features such as subwavelength waveguiding and frequency conversion capability with the ability to use optical tweezers to manipulate nanowires in realistic physiological environments can be used for various biological applications as local light and force sources (Fig. 12). Due to the subwavelength optical waveguiding nature of niobate nanowires, it is possible in combination with laser tweezers to create highly localized excitation source with the size which is determined by the nanowire tip diameter and to achieve the high spatial accuracy of nanowire position. This method is not only limited to the membrane proteins but it can be extended also to the imaging of intracellular compartments such as mitochondria. The success of intracellular imaging depends mostly on the nanowires functionalization with appropriate surface chemistries which will decrease the force required to introduce wires in the cell (Wallace \& Sansom, 2008).

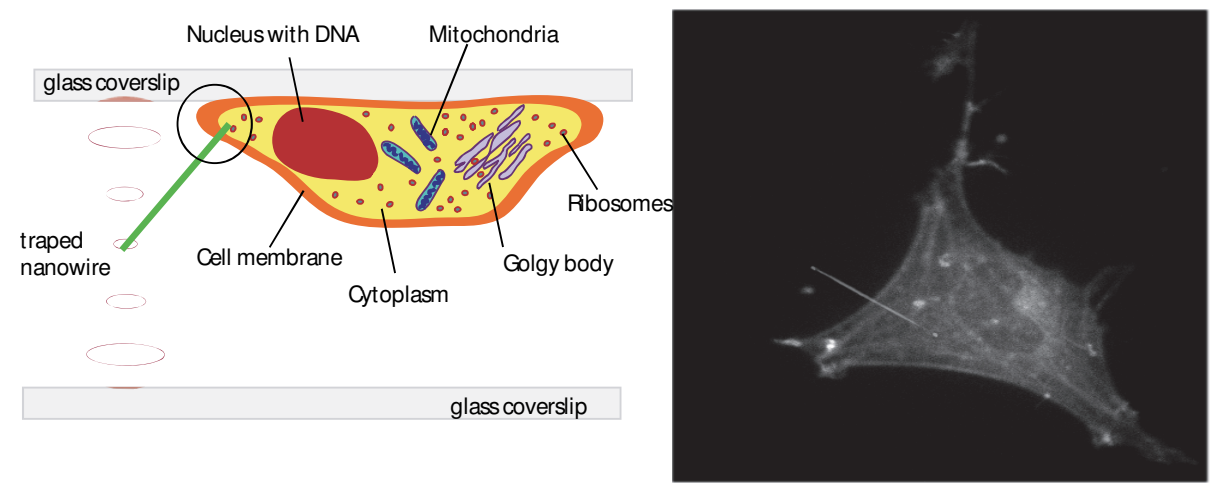

Fig. 12. Schematic of the experimental geometry where laser tweezers are used to accurately position the nanowire on the cell membrane while the nanowire through efficient SHG creates local excitation source. Fluorescence micrograph of a cultured mouse fibroblast (NIH 3T3) expressing YFP labeled ezrin and a nanowire.

\section{Applications in optofluidics environment}

In this section, we show applications of nanowires in an optofluidics environment. First we describe the devices fabrication, then a simulation of the device and finally experimental results with the nanowires.

The optofluidics device fabrication implies different process flows depending on the applications (Fig. 13). For dielectrophoresis applications, a $1 \mathrm{~mm}$ thick glass plate with a conductive electrode pattern in indium tin oxide (ITO) is used as described in (Choi et al., 2006). The sample is fabricated using contact photo lithography (Fig. 13 a). First, we obtain ITO coated glass plates rated at $30-60 \Omega$ /square. Next, positive photoresist is spincoated onto the plates. A pattern is exposed using a UV mask aligner and developed. Finally, the ITO is etched to create the electrode pattern. In case of microfluidic channels that are made of polydimethylsiloxane (PDMS), the fabrication uses the replica molding technique (Fig. 13 b). A master mold is produced through UV lithography on a silicon wafer. After trimethylchlorosilane (TMCS) treatment of the master mold for $5 \mathrm{~min}$, the PDMS is poured onto the mold with a $10: 1$ base-to-curing agent ratio. After curing in an oven at $80^{\circ} \mathrm{C}$ for 1 $\mathrm{h}$, the silicone is released from the mold. 


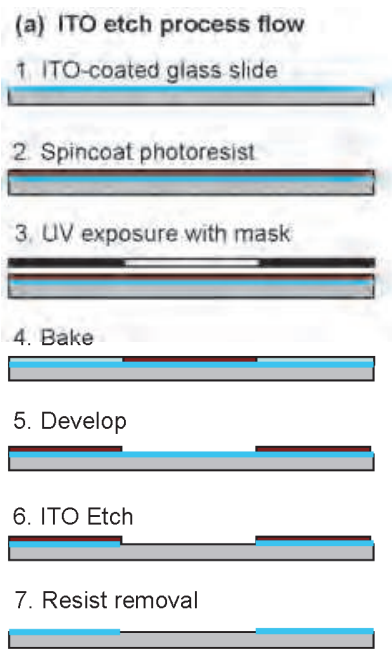

(b) PDMS process flow

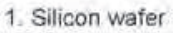

2 Spincoat photoresist

3. UV exposure with mask

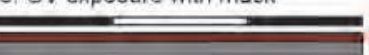

4. Bake

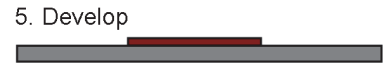

6. Surface treatment with TMCS

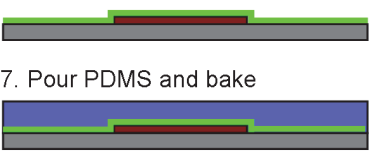

Fig. 13. (a) ITO process flow), (b) PDMS process flow.

For the first type of device, a floating dielectrophoresis electrode design was used to prevent shorting the nanowire after being trapped (Banerjee et al., 2007). This concept is best demonstrated by the COMSOL simulation (Fig. 14 a). A voltage is placed between the top and bottom electrodes. Due to the geometry of the floating electrodes, high electric fields are generated at the tips in the middle floating electrodes. The direction and magnitude of the electric field is indicated by the arrows. The high electric fields serve as dielectrophoretic traps and the direction of the electric field will align the nanowires due to electroorientation. For the specific design, triangular ITO electrodes were designed with a $2 \mu \mathrm{m}$ gap and the triangular ITO electrodes were separated by the contact electrodes with a $5 \mu \mathrm{m}$ gap. The application of an external electric field induces an additional electro-orientation force caused by dielectrophoresis (DEP). DEP forces have been utilized to orient and manipulate several different types of dielectric nanowires (Burke, 2004).

(a)

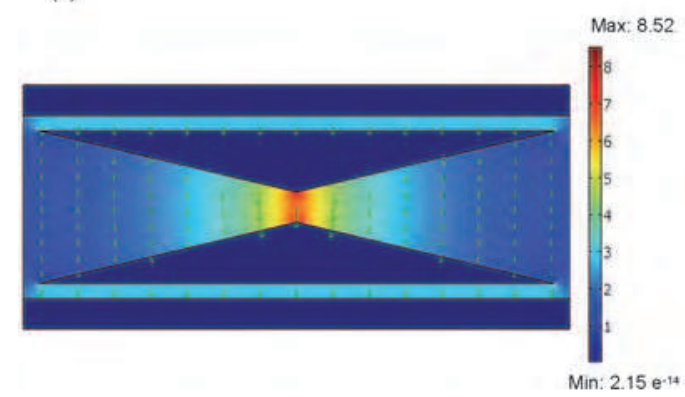

(b)

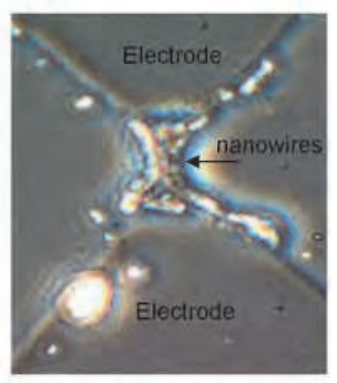

Fig. 14. (a) COMSOL simulation of the electrode design. Normalized surface electric field $(\mathrm{V} / \mathrm{m})$, arrow electric field $(\mathrm{V} / \mathrm{m}) .(\mathrm{b})$ White light image of the trapping of Lithium niobate nanowires at the tips of the floating electrodes. 
A standard ITO etch with a 5:6:1 ratio of $\mathrm{H}_{2} \mathrm{O}: \mathrm{HCl}(40 \%): \mathrm{HNO}_{3}(60 \%)$ at room temperature was utilized. By changing the etching time, we were able to control the size of the gap between the triangular electrodes. With a 5 minutes etch, the electrodes were shorted and there was no gap. At 10 minutes, a $1 \mu \mathrm{m}$ gap existed. By 15 minutes, a $2 \mu \mathrm{m}$ gap was formed. Results utilized a 10 to 15 minutes electrode etch. A PDMS microfluidic channel with the dimensions of $100 \mu \mathrm{m}$ wide, $10 \mu \mathrm{m}$ deep and $1.5 \mathrm{~cm}$ long was placed to overlap with the electrode gap. The microfluidic channel can hold $15 \mathrm{~nL}$ of liquid. To obtain at least a single nanowire in the entire channel, a concentration of $6.7 \times 10^{4}$ particles $/ \mathrm{mL}$ is required. Fig. 14 (b) shows the trapping of several nanowires with the floating electrodes design.

On top of the previous electrode device, we placed a PDMS chip with a single channel to orient the nanowires between the two electrodes (Fig. 15). The purpose of such design is to be able to detect electro-optic effects. Indeed, the bulk photovoltaic effect present in $\mathrm{LiNbO}_{3}$ might be of interest to generate locally an electric field under laser illumination. As shown on Fig. 15 (b), it was possible to concentrate nanowires at the electrodes tip.
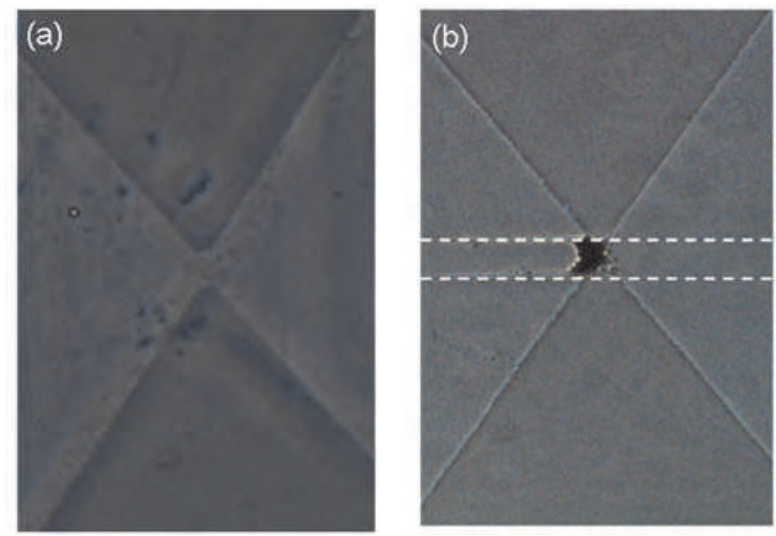

Fig. 15. (a) Empty electrodes (gap $=2 \mu \mathrm{m}$ width)(b) Electrodes with PDMS channel (indicated with dashed lines) to concentrate the nanowires at the tip.

The last device we developed was to manipulate lithium niobate nanowires in a fluidic environment and monitor the second-harmonic generation (SHG) response using an optical trapping setup (Fig. 16 left). The conductive electrode pattern is interdigitated with specific spacing and width that were previously determined (Choi et al., 2006). The optical tweezer is generated with a specific polarization that is determined with a half wave plate and located outside of the fluidic region, close to either substrate. Therefore, the nanowire is not allowed to orient along the direction of propagation and it locates itself orthogonal to the beam. This polarization imparts a force to orient the nanowire. Furthermore, an external electric field is applied to the nanowires using the pattern of electrodes. This external electric field induces an additional electro-orientation force caused by the dielectrophoretic (DEP) response of the nanowires. Note that the torque on the nanowire due to the external electric field is almost ten times greater in magnitude than the torque on the nanowire due to the optical polarization of the laser for the particle orientation denoted above. 

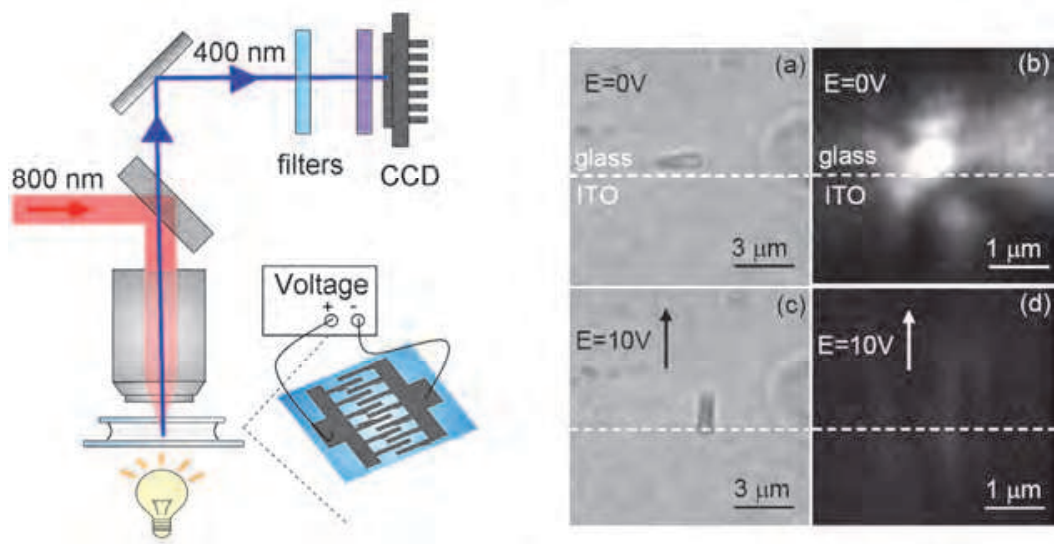

Fig. 16. Left: schematic of the setup for tweezing optically and electrically nanowires and detecting their SHG signal on a CCD after passing through filters to eliminate the incident light. Right: DEP response of a $\mathrm{LiNbO}_{3}$ nanowire suspended in $170 \mu \mathrm{S} / \mathrm{cm}$ conductivity deionized water ( $a$ and b) without and ( $c$ and d) with an electric field of $10 \mathrm{~V}_{\mathrm{pp}}$ at $150 \mathrm{kHz}$. (a) and (c) White light images; (b) and (d) SHG response (Reprinted with permission from (Grange et al., 2009). Copyright 2009, American Institute of Physics.)

Fig. 16 shows white light images (a and c) of a nanowire suspended in deionised water. At time $t=0$, no electric field ( $E_{\text {electrodes }}$ ) is applied and the nanowire is only tweezed by the laser $\left(E_{\text {laser }}\right)$ with a polarization parallel to the electrodes. Then a $10 V_{p p}$ electric field with a frequency of $150 \mathrm{kHz}$ is applied and after 1 second, the wire is oriented along this electric field due to the stronger field than the one from the laser. Fig. 16 (b and d) shows the SHG response measured with and without the applied electric field.

The nanowires are aligned with the field due to positive DEP forces. Increasing the conductivity of the suspension as well as changing the frequency of the applied electric field can change the type of DEP force on the nanowire which will result in the nanowire to not be aligned with the field. We notice the crossover conductivity between negative and positive DEP when the conductivity of the solution is around $170 \mu \mathrm{S} / \mathrm{cm}$. The combination provided by the applied external electric field and the polarization of the laser beam serves useful for several measurements in the nanometer scale. For instance, an estimation of the conductivity near the nanowire may be obtained. This may be useful if there is a conductivity gradient in the sample. Moreover, the polarization dependency of the nanowire allows for the detection of its position. The SHG signal can then be useful for detecting smaller wires close to the diffraction limit of a bright field microscope.

\section{Conclusion and outlook}

We described how most common types of alkaline niobates nanowires, $\mathrm{KNbO}_{3}, \mathrm{NaNbO}_{3}$ and $\mathrm{LiNbO}_{3}$ can be synthesized via hydrothermal or molten salt synthesis. We performed materials characterization to determine the composition of the end product of the synthesis as well as electron imaging to determine the aspect ratio of the three types of nanowires. As a further characterization step, we provided nonlinear optical measurements on single niobates nanowires with different setup geometries: transmission setup or optical tweezers. A SHG signal was measured for all types of nanowires as it is well known in similar non 
centrosymmetric bulk materials and it was possible to vary the SHG intensity by changing the polarization. Observed nonlinear properties of alkaline niobate nanowires suggest that all three types of nanowires could be used as frequency converters, mechano-optical probes or logic components at the nanoscale.

Then, plasmonics nanoshells were synthesized around $\mathrm{KNbO}_{3}$ nanowires. The thin gold layer serves, first, as enhancing the SHG signal through the plasmon resonance in the near infrared wavelength range of the excitation laser light. And secondly, it makes niobates nanowires compatible with biological entities and it can be an excellent starting point for further DNA or protein functionalization.

Several applications are described and performed to best use the nanowires properties at the overlap between the micro- and the nanoscale. Nanowires are demonstrated as trapped SHG probes for localized illumination. Using the polarization dependency of the SHG signal allow us to determine the orientation of nanowires in optofluidics environment too.

To conclude, applications using the alkaline niobates nanowires are only at the beginning and they are not yet using all the physical properties known for these perovskite materials. Therefore, there is room to improve the synthesis, for instance aspect ratio, to better know the physical properties and to be able to measure small signal as electro-optic effects on single nanowire.

\section{Acknowledgment}

We like to thank Demetri Psaltis, Jae-Woo Choi, Chia-Lung Hsieh, Ye Pu, Grégoire Laporte, Yioannis Papadopoulos, Arnaud Magrez, Anna Fontcuberta i Morral and Bernt Ketterer for helpful discussions and measurements.

\section{References}

An, C. H., Tang, K. B., Wang, C. R., Shen, G. Z., Jin, Y., \& Qian, Y. T. (2002). Characterization of $\mathrm{LiNbO}_{3}$ nanocrystals prepared via a convenient hydrothermal route. Materials Research Bulletin, Vol. 37, No. 11, pp. 1791-1796.

Atwater, H. A., \& Polman, A. (2010). Plasmonics for improved photovoltaic devices. Nature Materials, Vol. 9, No. 10, pp. 865-865.

Banerjee, S., White, B., Huang, L., Rego, B. J., O'Brien, S., \& Herman, I. P. (2007). Precise positioning of carbon nanotubes by ac dielectrophoresis using floating posts. Applied Physics a-Materials Science \& Processing, Vol. 86, No. 4, pp. 415-419.

Bhalla, A. S., Guo, R. Y., \& Roy, R. (2000). The perovskite structure - a review of its role in ceramic science and technology. Materials Research Innovations, Vol. 4, No. 1, pp. 3-26.

Boyd, R. W. (2008). Nonlinear Optics, Third Edition, Academic press, ISBN 978-0-12-369470-6 Burlington, Mass.

Burke, P. J. (2004). Nano-dielectrophoresis: Electronic Nanotweezers. In Encyclopedia of Nanoscience and Nanotechnology H. S. Nalwa (Ed.),Vol. 6, pp. 623. ISBN 1-58883-0012, Valencia, California.

Choi, J. W., Pu, A., \& Psaltis, D. (2006). Optical detection of asymmetric bacteria utilizing electro orientation. Optics Express, Vol. 14, No. 21, pp. 9780-9785.

Dadap, J. I. (2008). Optical second-harmonic scattering from cylindrical particles. Physical Review B, Vol. 78, No. 20, pp. 1098-0121.

Dadap, J. I., Shan, J., Eisenthal, K. B., \& Heinz, T. F. (1999). Second-harmonic Rayleigh scattering from a sphere of centrosymmetric material. Physical Review Letters, Vol. 83, No. 20, pp. 4045-4048. 
Fluck, D., \& Gunter, P. (2000). Second-harmonic generation in potassium niobate waveguides. Ieee Journal of Selected Topics in Quantum Electronics, Vol. 6, No. 1, pp. 122-131.

Grange, R., Choi , J.-W., Hsieh, C.-L., Pu, Y., Magrez, A., Smadja, R., Forro, L., \& Psaltis, D. (2009). Lithium niobate nanowires synthesis, optical properties, and manipulation. Applied Physics Letters, Vol. 95, No. 14, pp. 143105-143108.

Guo, Y. P., Kakimoto, K., \& Ohsato, H. (2004). Phase transitional behavior and piezoelectric properties of $\left(\mathrm{Na}_{0.5} \mathrm{~K}_{0.5}\right) \mathrm{NbO}_{3}-\mathrm{LiNbO}_{3}$ ceramics. Applied Physics Letters, Vol. 85, No. 18, pp. 4121-4123.

Hsieh, C.-L., Grange, R., Pu, Y., \& Psaltis, D. (2009). Three-dimensional harmonic holographic microcopy using nanoparticles as probes for cell imaging. Opt. Express, Vol. 17, No. 4, pp. 2880-2891.

Hsieh, C.-L., Pu, Y., Grange, R., \& Psaltis, D. (2010). Second harmonic generation from nanocrystals under linearly and circularly polarized excitations. Opt. Express, Vol. 18, No. 11, pp. 11917-11932.

Johnston, K. E., Tang, C. C., Parker, J. E., Knight, K. S., Lightfoot, P., \& Ashbrook, S. E. (2010). The Polar Phase of $\mathrm{NaNbO}_{3}$ : A Combined Study by Powder Diffraction, Solid-State NMR, and First-Principles Calculations. Journal of the American Chemical Society, Vol. 132, No. 25, pp. 8732-8746.

Ke, T. Y., Chen, H. A., Sheu, H. S., Yeh, J. W., Lin, H. N., Lee, C. Y., \& Chiu, H. T. (2008). Sodium niobate nanowire and its piezoelectricity. Journal of Physical Chemistry C, Vol. 112, No. 24, pp. 8827-8831.

Kong, L. B., Zhang, T. S., Ma, J., \& Boey, F. (2008). Progress in synthesis of ferroelectric ceramic materials via high-energy mechanochemical technique. Progress in Materials Science, Vol. 53, No. 2, pp. 207-322.

Li, L. H., Deng, J. X., Chen, J., Sun, X. Y., Yu, R. B., Liu, G. R., \& Xing, X. R. (2009). Wire Structure and Morphology Transformation of Niobium Oxide and Niobates by Molten Salt Synthesis. Chemistry of Materials, Vol. 21, No. 7, pp. 1207-1213.

Louis, L., Gemeiner, P., Ponomareva, I., Bellaiche, L., Geneste, G., Ma, W., Setter, N., \& Dkhil, B. Low-Symmetry Phases in Ferroelectric Nanowires. Nano Letters, Vol. 10, No. 4, pp. 1177-1183.

Magrez, A., Vasco, E., Seo, J. W., Dieker, C., Setter, N., \& Forro, L. (2006). Growth of singlecrystalline $\mathrm{KNbO}_{3}$ nanostructures. Journal of Physical Chemistry B, Vol. 110, No. 1, pp. 58-61.

Matthias, B. T., \& Remeika, J. P. (1949). Ferroelectricity in the Ilmenite Structure. Physical Review, Vol. 76, No. 12, pp. 1886-1887.

Nakayama, Y., Pauzauskie, P. J., Radenovic, A., Onorato, R. M., Saykally, R. J., Liphardt, J., \& Yang, P. D. (2007). Tunable nanowire nonlinear optical probe. Nature, Vol. 447, No. 7148, pp. 1098-1101.

Neuman, K. C., \& Block, S. M. (2004). Optical trapping. Rev. Sci. Instrum., Vol. 75, No. 9, pp. 2787-2809.

Niederberger, M., Pinna, N., Polleux, J., \& Antonietti, M. (2004). A general soft-chemistry route to perovskites and related materials: Synthesis of $\mathrm{BaTiO}_{3}, \mathrm{BaZrO}_{3}$, and $\mathrm{LiNbO}_{3}$ nanoparticles. Angewandte Chemie-International Edition, Vol. 43, No. 17, pp. 2270-2273.

Pribosic, I., Makovec, D., \& Drofenik, M. (2005). Formation of nanoneedles and nanoplatelets of $\mathrm{KNbO}_{3}$ perovskite during templated crystallization of the precursor gel. Chemistry of Materials, Vol. 17, No. 11, pp. 2953-2958.

Pu, Y., Grange, R., Hsieh, C.-L., \& Psaltis, D. (2010). Nonlinear optical properties of coreshell nanocavities for enhanced second-harmonic generation. Physical Review Letters, Vol. 104, No. 20, pp. 207401-207405. 
Saito, Y., Takao, H., Tani, T., Nonoyama, T., Takatori, K., Homma, T., Nagaya, T., \& Nakamura, M. (2004). Lead-free piezoceramics. Nature, Vol. 432, No. 7013, pp. 84-87.

Santulli, A. C., Zhou, H., Berweger, S., Raschke, M. B., Sutter, E., \& Wong, S. S. (2010). Synthesis of single-crystalline one-dimensional $\mathrm{LiNbO}_{3}$ nanowires. Crystengcomm, Vol. 12, No. 10, pp. 2675-2678.

Schwesyg, J. R., Eggert, H. A., Buse, K., Sliwinska, E., Khalil, S., Kaiser, M., \& Meerholz, K. (2007). Fabrication and optical characterization of stable suspensions of iron- or copper-doped lithium niobate nanocrystals in heptane. Applied Physics B-Lasers and Optics, Vol. 89, No. 1, pp. 15-17.

Shi, H., Li, X., Wang, D., Yuan, Y., Zou, Z., \& Ye, J. (2009). $\mathrm{NaNbO}_{3}$ Nanostructures: Facile Synthesis, Characterization, and Their Photocatalytic Properties. Catalysis Letters, Vol. 132, No. 1, pp. 205-212.

Shi, H. F., Li, X. K., Wang, D. F., Yuan, Y. P., Zou, Z. G., \& Ye, J. H. (2009). $\mathrm{NaNbO}_{3}$ Nanostructures: Facile Synthesis, Characterization, and Their Photocatalytic Properties. Catalysis Letters, Vol. 132, No. 1-2, pp. 205-212.

Shoji, I., Kondo, T., \& Ito, R. (2002). Second-order nonlinear susceptibilities of various dielectric and semiconductor materials. Optical and Quantum Electronics, Vol. 34, No. 8, pp. 797-833.

Sutherland, R. L. (2003). Handbook of Nonlinear Optics Hardcover Marcel Dekker, ISBN 0824794265, New York.

Wallace, E. J., \& Sansom, M. S. P. (2008). Blocking of carbon nanotube based nanoinjectors by lipids: A simulation study. Nano Letters, Vol. 8, No. 9, pp. 2751-2756.

Wang, G. Z., Selbach, S. M., Yu, Y. D., Zhang, X. T., Grande, T., \& Einarsrud, M. A. (2009). Hydrothermal synthesis and characterization of $\mathrm{KNbO}_{3}$ nanorods. Crystengcomm, Vol. 11, No. 9, pp. 1958-1963.

Wang, G. Z., Yu, Y. D., Grande, T., \& Einarsrud, M. A. (2009). Synthesis of $\mathrm{KNbO}_{3}$ Nanorods by Hydrothermal Method. Journal of Nanoscience and Nanotechnology, Vol. 9, No. 2, pp. 1465-1469.

Wang, L. H., Yuan, D. R., Duan, X. L., Wang, X. Q., \& Yu, F. P. (2007). Synthesis and characterization of fine lithium niobate powders by sol-gel method. Crystal Research and Technology, Vol. 42, No. 4, pp. 321-324.

Weber, M. J. (2003). Handbook of optical materials, CRC Press, ISBN 0849335124, New York.

Wood, B. D., Mocanu, V., \& Gates, B. D. (2008). Solution-Phase Synthesis of Crystalline Lithium Niobate Nanostructures. Advanced Materials, Vol. 20, No. 23, pp. 4552-4556.

Wooten, E. L., Kissa, K. M., Yi-Yan, A., Murphy, E. J., Lafaw, D. A., Hallemeier, P. F., Maack, D., Attanasio, D. V., Fritz, D. J., McBrien, G. J., \& Bossi, D. E. (2000). A review of lithium niobate modulators for fiber-optic communications systems. Ieee Journal of Selected Topics in Quantum Electronics, Vol. 6, No. 1, pp. 69-82.

Wu, S. Y., Liu, X. Q., \& Chen, X. M. (2010). Hydrothermal synthesis of $\mathrm{NaNbO}_{3}$ with low $\mathrm{NaOH}$ concentration. Ceramics International, Vol. 36, No. 3, pp. 871-877.

Zhao, L. L., Steinhart, M., Yosef, M., Lee, S. K., \& Schlecht, S. (2005). Large-scale templateassisted growth of $\mathrm{LiNbO}_{3}$ one-dimensional nanostructures for nano-sensors. Sensors and Actuators B-Chemical, Vol. 109, No. 1, pp. 86-90.

Zhu, H. Y., Zheng, Z. F., Gao, X. P., Huang, Y. N., Yan, Z. M., Zou, J., Yin, H. M., Zou, Q. D., Kable, S. H., Zhao, J. C., Xi, Y. F., Martens, W. N., \& Frost, R. L. (2006). Structural evolution in a hydrothermal reaction between $\mathrm{Nb}_{2} \mathrm{O}_{5}$ and $\mathrm{NaOH}$ solution: From $\mathrm{Nb}_{2} \mathrm{O}_{5}$ grains to microporous $\mathrm{Na}_{2} \mathrm{Nb}_{2} \mathrm{O}_{6}{ }_{2} /{ }_{3} \mathrm{H}_{2} \mathrm{O}$ fibers and $\mathrm{NaNbO}_{3}$ cubes. Journal of the American Chemical Society, Vol. 128, No. 7, pp. 2373-2384. 


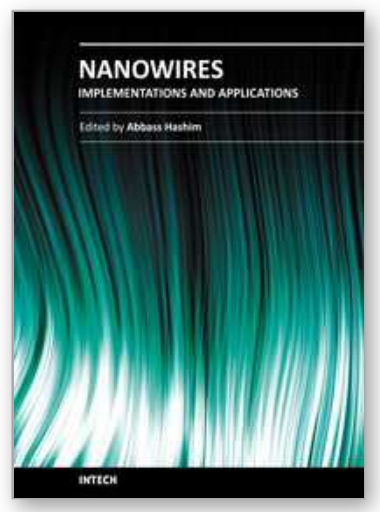

\author{
Nanowires - Implementations and Applications \\ Edited by Dr. Abbass Hashim
}

ISBN 978-953-307-318-7

Hard cover, 538 pages

Publisher InTech

Published online 18, July, 2011

Published in print edition July, 2011

This potentially unique work offers various approaches on the implementation of nanowires. As it is widely known, nanotechnology presents the control of matter at the nanoscale and nanodimensions within few nanometers, whereas this exclusive phenomenon enables us to determine novel applications. This book presents an overview of recent and current nanowire application and implementation research worldwide. We examine methods of nanowire synthesis, types of materials used, and applications associated with nanowire research. Wide surveys of global activities in nanowire research are presented, as well.

\title{
How to reference
}

In order to correctly reference this scholarly work, feel free to copy and paste the following:

Rachel Grange, Fabrizia Dutto and Aleksandra Radenovic (2011). Niobates Nanowires: Synthesis, Characterization and Applications, Nanowires - Implementations and Applications, Dr. Abbass Hashim (Ed.), ISBN: 978-953-307-318-7, InTech, Available from: http://www.intechopen.com/books/nanowiresimplementations-and-applications/niobates-nanowires-synthesis-characterization-and-applications

\section{INTECH}

open science | open minds

\section{InTech Europe}

University Campus STeP Ri

Slavka Krautzeka 83/A

51000 Rijeka, Croatia

Phone: +385 (51) 770447

Fax: +385 (51) 686166

www.intechopen.com

\section{InTech China}

Unit 405, Office Block, Hotel Equatorial Shanghai

No.65, Yan An Road (West), Shanghai, 200040, China

中国上海市延安西路65号上海国际贵都大饭店办公楼 405 单元

Phone: $+86-21-62489820$

Fax: +86-21-62489821 
(C) 2011 The Author(s). Licensee IntechOpen. This chapter is distributed under the terms of the Creative Commons Attribution-NonCommercialShareAlike-3.0 License, which permits use, distribution and reproduction for non-commercial purposes, provided the original is properly cited and derivative works building on this content are distributed under the same license. 